\title{
Motivating contributors in social media networks
}

\author{
Vivek K. Singh \\ University of California, Irvine \\ singhv@uci.edu
}

\author{
Ramesh Jain \\ University of California, Irvine \\ jain@ics.uci.edu
}

\author{
Mohan S. Kankanhalli \\ National University of \\ Singapore \\ mohan@comp.nus.edu.sg
}

\begin{abstract}
Despite recent advancements in user-driven social media platforms, tools for studying user behavior patterns and motivations remain primitive. We highlight the voluntary nature of user contributions and that users can choose when (and when not) to contribute to the common media pool. We use a Game theoretic framework to study the dynamics of a social media network wherein contribution costs are individual but gains are common. We model users as rational selfish agents, and consider domain attributes like voluntary participation, virtual reward structure and public-sharing to model the dynamics of this interaction. The created model describes the most appropriate contribution strategy from each user's perspective. Next, we consider the problem of mechanism design from a system designer's perspective who is interested in finding the optimal incentive levels to influence the selfish end-users so that the overall system utility is maximized. We demonstrate how a system administrator can exploit the selfishness of its users, to design incentive mechanisms which help in improving the overall task completion probability and system performance, while possibly still benefiting the individual users.
\end{abstract}

\section{Categories and Subject Descriptors}

H.1.2 [Information Systems]: Models and principles, User/ Machine systems

\section{General Terms}

Human factors

\section{Keywords}

Motivation, social media, user behavior, game theory

\section{INTRODUCTION}

With the emergence of Web 2.0 and multiple related social media applications like Flickr, Youtube, Facebook, Wikipedia

Permission to make digital or hard copies of all or part of this work for personal or classroom use is granted without fee provided that copies are not made or distributed for profit or commercial advantage and that copies bear this notice and the full citation on the first page. To copy otherwise, to republish, to post on servers or to redistribute to lists, requires prior specific permission and/or a fee.

WSM'09, October 23, 2009, Beijing, China.

Copyright 2009 ACM 978-1-60558-759-2/09/10 ...\$5.00. etc., research interest has grown in multiple aspects of social media including data sharing, image tagging, mashups, ontologies, retrieval etc. While these contributions have significantly advanced the state of the art from the technology perspective, not much research attention has been given till now to the end-user or social aspect of social media research. Despite significant interest in concepts like crowd-sourcing [8], collective intelligence [11], human-computation [19] etc., the tools to undertake user behavior analysis in social media networks are still in their infancy and no theoretical frameworks are available to mathematically analyze why and how often do users contribute to such social media?

That notwithstanding, social media networks are becoming increasingly relevant each day. Citizen-journalists are already providing interesting event information and images for common benefit to various news agencies[3]. Similarly multiple users are already using tools like Google Image Labeler[1] to tag images and contributing content to Wikipedia[4], thus creating a rich collective information mechanism which can provide common benefits to a larger society.

An important point to consider in all these applications is that the user contribution is totally voluntary. Further the decision making is completely distributed and there are no means for central coordination or explicit communication between the various participating users. This brings us to the important issue of user motivation and that the individual users will contribute to such social media networks only based on their personal utility decisions.

Such a setup, though compelling, leads to multiple conflicting goals. While, the task completion costs are incurred by the individuals, the benefits are common. Thus while the owners of systems such as New York Times, Google Labeler, Wikipedia etc. are interested in maximizing the tasks accomplished, individual agents may be interested in maximizing their personal utility gain in such a sensing/contentprovision mechanism. A key question which arises in such a scenario is how can an individual user optimally decide his/her contribution strategy i.e. when (and when not) should he/she undertake the social media task. A system administrator on the other hands is interested in finding the optimal incentive levels to influence these selfish end-users so that the overall system utility is maximized.

Clearly, there are no currently available tools which can answer such questions. The analysis and answer to such questions requires explicit modeling of user behavior as well as considering the specific characteristics of the domain being considered. Hence we propose the use of a game theoretic framework, which models users as rational (selfish) agents 


\begin{tabular}{|c|c|c|c|c|c|}
\hline Case & Participation & Taxation & Reward currency & Career benefits & Usage pattern \\
\hline Social media & Voluntary & "Not enforceable & $\overline{\overline{\text { Virtual }}}$ & Rare & $\overline{\text { public-good }}$ \\
\hline Physical societies & Mandatory & Enforceable & Real & - & - \\
\hline $\begin{array}{l}\text { Open-source software \& } \\
\text { scientific contributions }\end{array}$ & Voluntary & - & - & Yes & $\begin{array}{l}\text { Partially } \\
\text { regulated }\end{array}$ \\
\hline P2P/ networking & Voluntary & - & Virtual & - & $\begin{array}{l}\text { Monitored \& } \\
\text { regulated }\end{array}$ \\
\hline
\end{tabular}

Table 1: Distinct characteristics of different type of contribution mechanisms

and incorporates the dynamics of social media (e.g. voluntary participation, virtual reward structure and publicsharing) to gain some insights/ explanations for user behavior patterns and also prescriptive guidelines for system designers to motivate their users.

We demonstrate how game theoretic modeling can be used to answer the above-mentioned questions. We study the user-user interaction and show how a user can find her optimal contribution level. Further, we demonstrate how a system designer can draw insights from such behavior patterns and exploit the selfishness of its users, to design incentive mechanisms which help in improving the overall system performance, while possibly still benefiting the individual users.

\section{RELATED WORK AND DOMAINS}

\subsection{Why are social media networks different?}

Similar problems have been studied in multiple contexts in the past. From an economics perspective, the problem of 'public goods' [13] and optimal taxation [6] is well studied. However, emerging web-based 'societies' are fundamentally different as participation in them is voluntary and as such no taxation can be enforced. Only incentives, if appropriate can be given. Further, the reward on these social media sites etc. is typically a 'virtual currency' which has very different dynamics than real money. Such virtual currency (e.g. reputation points, extra bandwidth, virtual weaponry, gadgets etc.) is like 'fairy gold-dust' and typically costs the system designers exponentially less than their perceived value from user perspective. While some of this virtual currency is starting to be traded by users for real-world money [17], the marginal cost for system designers to grant such currency remains very low.

The issue of selfishness and contributions to a society also differ from scientists, and open-source software developers, as their 'contributions' are typically in-sync with their full time vocation. Hence they have direct and indirect professional benefits (grants, citations, downloads, jobs) from demonstrating their skill level. While a small percentage of contributors in media networks (e.g. directors on Youtube, or photographers on Flickr) might generate some career benefits from their contributions, these benefits remain atypical. More frequently the contributions to social media sites like Google Image Labeler, Photo-synth [2], Wikipedia do not involve rights and recognition as is common in open-source or scientific communities.

Lastly, social media network paradigms also differ from $\mathrm{P} 2 \mathrm{P}$ or network routing disciplines as you cannot really calculate or regulate the usage characteristics. The produced content is truly a public-good [13] for everybody like 'sunshine'.

Thus social media contributions deal with a unique set of parameters involving, voluntary participation, no taxation, real cost, virtual incentives, rare career benefits, and no regulations on usage characteristics. In this work we consider these differences in modeling and studying the media networks. Table 1 provides a summary of the comparison between different related scenarios and how social media contributions differs from each of them.

\subsection{Related work in social media}

There have been attempts at enhancing user experience, and using that to get social media related tasks undertaken. Human-computing work by Von Ahn [19] is an excellent example of this.

Works like [12], study user behavior patterns in terms of the way they interact with social media sites (Youtube). They classify the users into different categories based on their access patterns, comment frequency, subscriptions etc. These are very important studies from the perspective of understanding general user behavior patterns. We maintain our focus in this paper though on user behavior from a motivation perspective and on incentivizing user contributions for better system performance.

Other works study the motivation of contributors on MovieLens, Wikipedia etc. from a sociology or psychological perspective $[7,16,15]$. Schroer et al. [15] discuss the intrinsic and extrinsic motivations for German Wikipedia contributors. Works like $[7,16]$ have highlighted how different forms of motivation (e.g. locked-out tools/ features in Slash-dot, extra weapons in World-of-Warcraft forum and comparative reputation/status within community etc.) can be used to motivate users. However, all these studies are 'qualitative' and aimed at identifying what type of motivations work well in on-line communities. We however aim to provide 'quantitative' mechanisms to find out how many such extra features, weapons, or how much (e.g. extra bandwidth, reputation points etc.) are most suitable for different scenarios.

Mechanism design (i.e. defining rules of a game to achieve certain outcomes) is an area of growing importance (including recent Nobel prizes) in economics. It is also slowly making inroads into on-line communities (e.g. for creating optimal reputation feedback mechanisms in eBay like auction scenarios [5]).

\section{BACKGROUND: GAME THEORY}

Game theory is a branch of applied mathematics that is used in the social sciences (most notably economics), biology, engineering, political science, international relations 
and is becoming increasingly relevant in computer science. It is used to mathematically capture behavior in strategic situations, in which an individual's utility from choices undertaken depends on the choices made by others. Thus it is often used for studying optimality and stable points in multi-agent problems, as opposed to conventional operation research or calculus based approaches which are well-suited for single agent optimizations.

Here, we provide a quick refresher for some of the gametheoretic terms and concepts as relevant to this work.

1. Game: A game refers to any situation wherein multiple (2 or more) agents are making strategy decisions, and the chosen strategy effects the utility obtained by that agent as well as the other agents involved. Games can be zero-sum, wherein one agent's loss is considered other agent's gain e.g. war-like situations, or nonzero-sum, where one agent's loss does not necessarily means others gain. In our formalisms the users (among themselves in section 4) and the users and system designers (in section 5) are both playing non-zero-sum games.

2. Nash equilibrium: Nash equilibrium is a solution concept in game theory which defines a point where each agent knows other agent's strategy options, and from which no agent has anything to gain by changing only her own strategy unilaterally. It is useful in giving guaranteed utility bounds to users, as once they choose their strategy based on the Nash equilibrium, it is in other agent's benefit to respond with their Nash equilibrium response. Any other response can only decrease their utility. Please note that multiple Nash equilibria may exist in the same game and agents can move or converge bilaterally to another Nash equilibrium (especially in repeated games).

3. Mixed Strategy Nash equilibrium (MSNE): A mixed strategy Nash equilibrium is a probabilistic variant of Nash equilibrium wherein the agents do not fix themselves to a single strategy but rather decide on the appropriate mixture of strategies which guarantees that no agent can gain anything by unilaterally diverting from it. Pure Nash equilibriums can be considered to be boundary cases of mixed strategy Nash equilibria.

\section{A GAME THEORETIC FRAMEWORK: THE USER VIEWPOINT}

In this section we model user-user interaction patterns, and describe how rational (selfish) users may make optimal contribution decisions.

\subsection{Problem motivation}

To motivate and ground the problem to a real life scenario from start, let us consider a citizen-journalism task $(T)$ where a 'suspicious bag' left unattended at a train station can be reported by any of the $N$ persons (agents) walking past. The gain $(G)$ is common but the cost incurred $\left(c_{i}\right)$ is individual. The problem from an agent $(i)$ 's perspective is to find the percentage of times which he/she should report the bag himself/herself.

\begin{tabular}{|c|c|c|}
\hline Agent $1^{\text {OtherAgent }}$ & Do & Don't \\
\hline Do & $G-c, G-c$ & $G-c, G$ \\
\hline Don't & $G, G-c$ & 0,0 \\
\hline
\end{tabular}

Table 2: Game between 2 users: matrix showing net utility for each user, under different strategies adopted by each player.

\begin{tabular}{|c|c|c|}
\hline Agent $_{i}$ OtherAgents & Do & Don't \\
\hline Do & $G-c_{i}$ & $G-c_{i}$ \\
\hline Don't & $G$ & 0 \\
\hline
\end{tabular}

Table 3: Game between user $\left(\right.$ Agent $\left._{i}\right)$ and others: matrix showing net utility for $A_{g e n t}{ }_{i}$, under different strategies adopted.

\subsection{Problem formulation}

Let there be $N$ social agents which can undertake a common task $T$. Let the cost for an agent $i$ to undertake task $T$ be $c_{i}$. While the costs are individual, the gains incurred $(G)$ are taken to be common i.e. everybody gains equally. Each user can incur gain $G$, from a task if either it completes the task, or it does not but somebody else completes it. Hence, the net utility of each agent $E U_{i}$ is a function of it's cost, gain, its chosen strategy in terms of how often to undertake the task $\left(P_{i}\right)$, as well as the probability of task being undertaken by (at least one of the) other agents $\left.\left(P_{D o}^{A l l-\{i\}}\right)\right)$. Hence, the problem from each agent's perspective is to find its best response strategy, (i.e. probability of doing task) which maximizes its net utility.

$$
\underbrace{\operatorname{argmax}}_{P_{i} \in[0,1]} E U_{i}=f\left(c_{i}, G, P_{i}, P_{D o}^{A l l-\{i\}}\right)
$$

The provided formulation incorporates two important characteristics of social media networks. Voluntary participation characteristic is innately represented in the problem statement, and the property of common-gain once the task is completed is also made explicit.

In the formulation presented here, we assume that the users are selfish agents, that there is no collusion or agreement between them, and that the cost incurred is positive and less than gain $(0<c \leq G)$. Also, in this formulation we use the term 'social media task', generically to include all relevant scenarios like those involving photo/video sharing, image tagging, commenting, content provision, linking, or content flagging. Lastly, we use 'agent' as a neutral term to represent any contributor who can undertake the relevant task.

\subsection{Approach}

We employ a game theoretic framework to solve Eq. 1. To solve this problem, we start with a simple two person nonzero sum game to study the interaction between two homogeneous agents and then iteratively add more complexities.

\subsubsection{Two agents, 1 social media task}

As shown in table 1 , for agent 1 there are two possible strategy options. $\mathrm{He} / \mathrm{she}$ can either 'do' the the task or 'dont' do it. If he/she chooses to do the task and the other 
agent also undertakes the task, then both will get the Gain $G$ but also incur the cost $c$. Agent 1's best case scenario is when he/she does not undertake the task but the other agent does it. Hence agent 1 will receive gain $G$ without any cost while other agent will incur it. However, if both agents choose the 'dont' strategy, then there will be no gain achieved as the task is not undertaken.

The solution concept used in such settings where other agent's decisions affect your utilities is Nash equilibrium. The Nash equilibrium occurs when none of the agents can unilaterally move to a better rewarding state[14]. In this particular scenario, both the agents have a choice to either use the strategy 'Do' or 'Dont' and there exist 3 Nash equiliria. Two pure Nash equilibria exist at states [Do, Don't] and [Dont, Do] for agents 1 and 2 respectively. However, they favor one agent or the other and are unlikely to be maintained in long term. Hence, a mixed strategy solution seems a stable long term solution. The mixed strategy Nash equilibrium guarantees that:

$P_{i}^{*} \in[0,1], P_{i} \neq P_{i}^{*}: E U_{i}\left(P_{i}^{*}, P_{D o}^{A l l-\{i\}^{*}}\right) \geq E U_{i}\left(P_{i}, P_{D o}^{A l l-\{i\}^{*}}\right)$

where:

$P_{i}^{*}$ is the optimal strategy for agent $i$, and $P_{D o}^{A l l-\{i\}^{*}}$ represents the cumulative effect of best possible strategy choices made by other agent(s).

A mixed strategy Nash equilibrium (MSNE) can be computed based on the condition of choice indifference i.e. when the agents do not gain (or lose) by changing their strategies [14]. This makes for a good equilibrium point because if the agents prefer one choice, obviously they shall go for the better choice. Thus the equilibrium needs to take place at a point where both agents do not stand to gain (or loose) any value by strategy selection.

In the given scenario (table 2), if we equate the two options for (say) player 1. We get:

$$
p \cdot(G-c)+(1-p) \cdot(G-c)=p \cdot(G)+(1-p) \cdot 0
$$

where:

$p$ is the probability of agent 2 choosing strategy 1 i.e. Do. This gives the value of $p$ as:

$$
p=\frac{G-c}{G}
$$

For heterogeneous agents it changes to:

$$
p_{2} \cdot\left(G-c_{1}\right)+\left(1-p_{2}\right) \cdot\left(G-c_{1}\right)=p_{2} \cdot(G)+\left(1-p_{2}\right) \cdot 0
$$

where $p_{2}$ is the probability of agent 2 choosing strategy 1 i.e. Do. This gives the value of $p_{2}$ as:

$$
p_{2}=\frac{G-c_{1}}{G}
$$

and similarly $p_{1}$ is :

$$
p_{1}=\frac{G-c_{2}}{G}
$$

If each agent chooses to undertake the task with just $p_{1}$ (resp. $p_{2}$ ) probability, s/he will get the same net utility as doing the task always by him/her self.

\subsection{2 $\quad N$ agents, 1 social media task}

For the heterogeneous, $N$ agent case let us look again at table 2. For a Nash Equilibrium to exist the two strategy options for agent $i$ must provide same net utility. Thus:

$$
G-c_{i} \cdot\left(P_{D o}^{A l l-\{i\}}\right)+G-c_{i} \cdot\left(1-P_{D o n^{\prime} t}^{A l l-\{i\}}\right)=P_{D o}^{A l l-\{i\}} \cdot G+0
$$

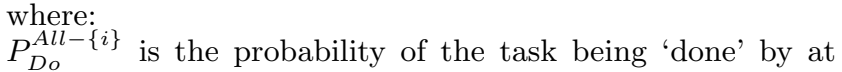
least one of the $N-1$ agents left after removing the $i^{\text {th }}$ agent from the set of 'all' agents.

Using the above equation, the equilibrium probability of the task being 'not done' by any of the other agents can be calculated as:

$$
P_{D o n^{\prime} t}^{A l l-\{i\}}=\frac{c_{i}}{G}
$$

Similar equations can be formulated for all values of $i$.

$$
\begin{aligned}
& P_{D o n^{\prime} t}^{A l l-\{1\}}=\frac{c_{1}}{G} \\
& P_{D o n^{\prime} t}^{A l l-\{2\}}=\frac{c_{2}}{G} \\
& \vdots \\
& P_{D o n^{\prime} t}^{A l l-\{N\}}=\frac{c_{N}}{G}
\end{aligned}
$$

Combining (multiplying) all of these equations gives us:

$$
\left\{\overline{P_{1}} \cdot \overline{P_{2}} \cdot \ldots \overline{P_{N}}\right\}^{N-1}=\prod_{i=1}^{N} \frac{c_{i}}{G}
$$

where:

$\overline{P_{i}}$ is the probability of the task not being done by agent $i$. Thus,

$$
P_{D o n^{\prime} t}^{A l l}=\sqrt[N-1]{\prod_{i=1}^{N} \frac{c_{i}}{G}}
$$

or:

$$
P_{D o}^{A l l}=1-\sqrt[N-1]{\prod_{i=1}^{N} \frac{c_{i}}{G}}
$$

and combining Eq. 9 and Eq. 12 gives us the optimal contribution strategy i.e. equilibrium probability for the agent $i$ to 'not' undertake the task as follows:

$$
\overline{P_{i}}=\sqrt[N-1]{\prod_{i=1}^{N} \frac{c_{i}}{G}} \times \frac{G}{c_{i}}
$$

Note that the feasibility condition of a solution in which agent $i$ must participate in the task, the condition is that:

$$
\frac{c_{i}}{G} \geq \sqrt[N-1]{\prod_{i=1}^{N} \frac{c_{i}}{G}}
$$

and that for homogeneous case (if applicable) the above equation reduces to:

$$
\overline{P_{i}}=\left(\frac{c}{G}\right)^{\frac{1}{N-1}}
$$

\subsection{First insights}

To illustrate the basic concepts let us consider how the model and the derived solution works for a simple scenario 


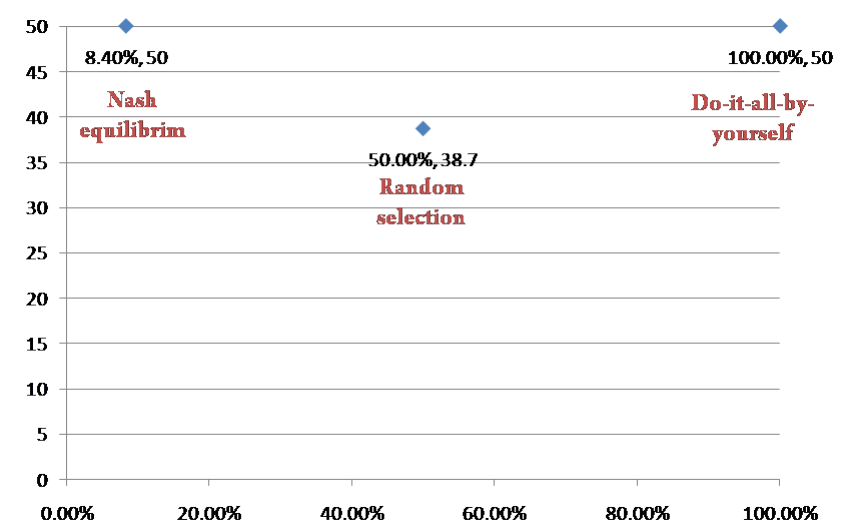

Figure 1: Effect of different task completion strategies upon Agent 1's utility

of the unattended bag reporting where we have 3 agents each with a cost of 50,60 and 70 respectively while the common gain from reporting is 100 . Thus using the parameter values $\left(c_{1}=50, c_{2}=60, c_{3}=70, G=100\right)$ in Eq. 16, we get the equilibrium probabilities for each agent to undertake the task to be $0.084,0.346$ and 0.445 respectively. The utility for each agent was found to decrease if it moved away from the equilibrium point. It was also interesting to note that in spite of doing the task much lesser times, the agents obtained the same utility (values 50, 40, 30 resp.) as they would have achieved by doing the task always by themselves. This is shown in figure 1, for Agent 1, who at MSNE, made a net gain of 50 , by contributing just $8.4 \%$ times. Not changing the other agents response, this utility is the same as that obtainable by a naive 'do-it-all-by-yourself' approach which involves $100 \%$ contribution rate, and is more than that obtainable by random selection which involves $50 \%$ contribution rate.

We next proceeded to model the dynamics of a game scenario wherein a large number of selfish users are considering a common task (c=60, $\mathrm{G}=100, N \in[1,100])$. Upon varying the gain and costs we found the expected results of each user's utility increasing if the gain was high and decreasing if the cost was high. Since each user was selfishly guarding his/her incentives the utility of each agent was unaffected by the change in $N$.

However, the change in $N$ had a dramatic impact on the percentage of times the common task was completed. As can be seen from fig. 2, the task completion probability decreases (from 1.0 to 0.4 ) as $N$ increases. This was an interesting observation as intuitively one thinks that the probability of task getting done should increase with $N$, because if we multiply individual probabilities for everyone not doing the task, the overall probability of all not doing the task should be extremely low.

However, as the game theoretic model makes explicit, the knowledge about large $N$, makes each agent adjust its taskcompletion probabilities in such a way which guards its individual utilities but can bring down the overall task completion. It reminds us of the apathy which can exist in large groups of selfish individuals undertaking common tasks. In hind-sight it also resonates well with how the 'free-rider' [10] problem is non-existent in single person teams. In n-person

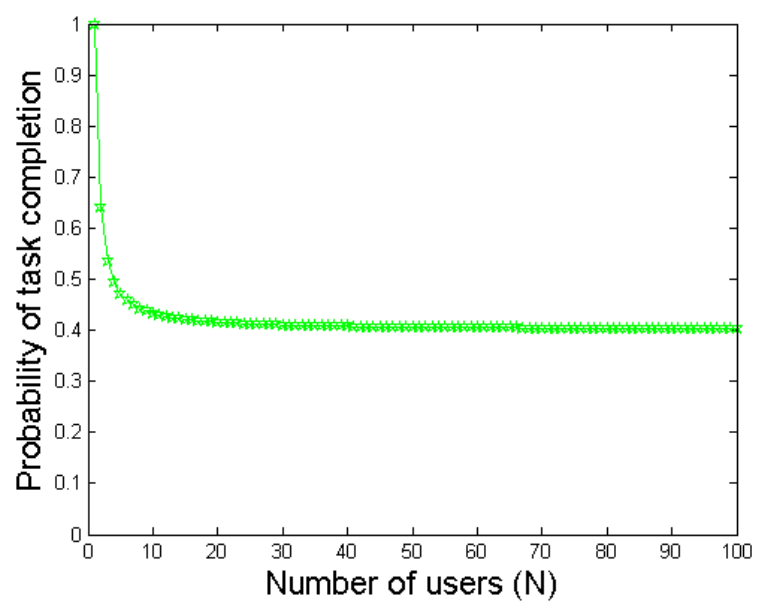

Figure 2: Effect of large number of users on task completion probability

teams (especially non-coordinated teams), there is always a finite possibility of everybody deciding to free-ride on a particular task.

This is quite alarming from a rational system-designers perspective.

\section{THE DESIGNER'S VIEWPOINT}

After studying the dynamics of user-user interaction and how a rational user would behave in such systems, let us use that as a building block towards studying dynamics of system-user interaction. We demonstrate how the system can exploit the features of user behavior to study the overall system performance, and in turn maximizing it's performance.

\subsection{Problem formulation}

Let us consider a case where the system-designer has an interest in maximizing the probability of task completion. Let her benefit from each task completed be $G_{s}$. Hence, without any mechanism design the net utility obtained by the system designer is $\left(P_{\text {NoBonus }}^{\text {All }} \cdot G_{s}\right)$, where $P_{\text {NoBonus }}^{\text {All }}$ is as found in previous section (Eq. 13). However, using mechanism design, the system designer can enhance this performance.

Let us assume that the system designer is open to granting an extra benefit $b$ to each user completing the task, so as to influence the users' (selfish) decision process of choosing how often to undertake the task. Clearly, providing the extra bonus $b$, does entail some additional cost on the system. However, as per the dynamics of social media systems, such benefits are typically 'virtual' (e.g. granting 'additional bandwidth', 'enhanced weaponry', 'titles/badges' or 'memorabilia'), and cost the system designer exponentially less than their perceived benefit by the user ${ }^{1}$.

For the current discussion, let us consider system cost for providing this additional bonus to be $C_{b}=\alpha \cdot b^{\frac{1}{\beta}}$, where $\alpha$, $\beta$ can be chosen based on the domain. ${ }^{1}$ The use of perceived changes in games to try and influence
agent interaction is well studied under hyper-game theory [18] 
Thus the overall utility problem for system designer is:

$$
\underbrace{\operatorname{argmax}}_{b \in[0, c]} E U_{s}=\left(P_{\text {Bonus }}^{\text {All }}\right) \cdot G_{s}-\sum_{i=1}^{N} P_{i, \text { Bonus }} \cdot C_{b}
$$

Thus the system designer's problem is to choose the optimal bonus $b$, such that overall system utility $E U_{s}$ is maximized. $E U_{s}$ will be maximized when a large increase is observed in probability of task completion due to the extra bonus, but the bonus cost is still low. The maxima will clearly lie at a trade-off point between these two components.

Note that the above formulation considers the distinct characteristics of social media systems as we consider voluntary participation, common gain amongst all users, stay away from taxation, and consider virtual reward currency which typically costs exponentially less to the system.

\subsection{Approach}

To quantify these parameters let us make simplifying assumptions of homogeneous costs and $C_{b}=b^{\frac{1}{2}}$ (i.e. $\alpha=1$ and $\beta=2$ ).

Based on extensions of the ideas already discussed under user-user interaction in sec. 4.3, the various parameters involved in Eq. 17 can be computed as follows.

Extension of Eq. 16 defines the probability of task completion (with bonus) for agent $i$.

$$
P_{i, \text { Bonus }}=1-\left(\frac{c-b}{G}\right)^{\frac{1}{N-1}}
$$

Similarly, extension of Eq. 12 can be used to compute the probability of overall task completion after bonus incentive.

$$
P_{\text {Bonus }}^{\text {All }}=1-\left(\frac{c-b}{G}\right)^{\frac{N}{N-1}}
$$

Now that we have the values for all the parametric components of Eq. 17, its solution can be computed using the standard calculus maximization methods or by choosing maxima as obtained by numerical methods.

\subsection{Case study}

To study the applicability of the proposed approach we undertook another simple case study. We considered a scenario with $N=1000$, the uniform $\operatorname{cost} c=60$, user and system gains as $G=100$ and $G_{s}=50$. We varied the bonus provided to the users in the approved range $b \in[0,60]$ (see fig. 3), and found the system's utility is maximized at $b=42$. Needless to say, similar values can be obtained for other scenarios too by simple parameter changes.

The net system utility for this task was found to be 29.87, which is higher than the value of 20 , as obtained without mechanism design.

As can be seen from fig. 4, the probability of the task being undertaken by at least one of the $N$ users, increases to around 0.82 (red colored plot) with the use of this additional incentive value. This is as opposed to the 0.40 probability without the incentive mechanism (green plot, also see fig. 2 ). The value of net utility for the homogeneous players was also found to increase to 82 (from 40.0 without bonus).

Hence the game-theoretic framework was indeed useful in finding an optimal level of incentive level which maximizes the system's net utility and increases the probability of taskcompletion while also enhancing the net utility of each user.

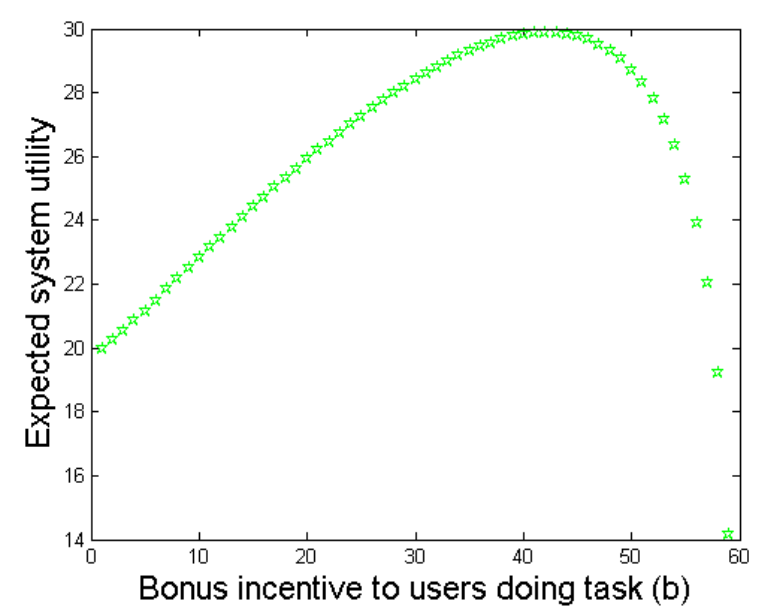

Figure 3: System Utility Vs Bonus incentive

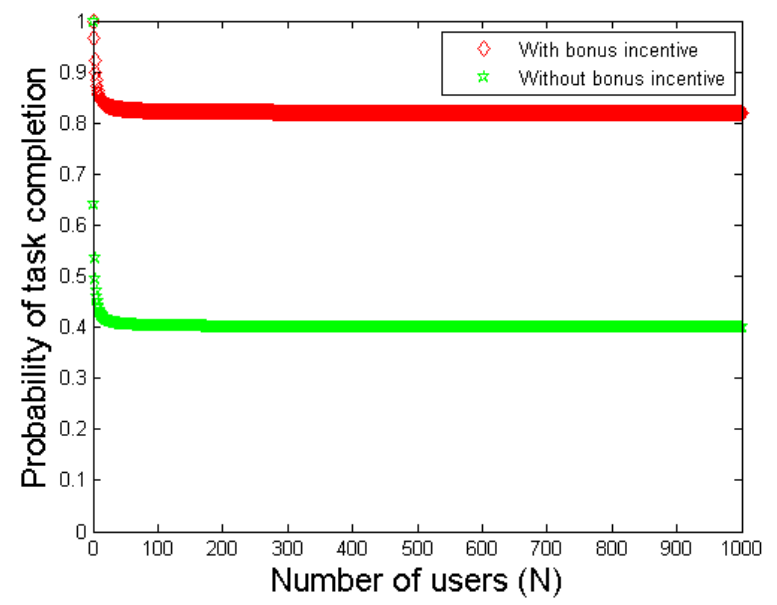

Figure 4: Effect of bonus on task completion probability 


\section{DISCUSSION AND FUTURE WORK}

We realize that the framework discussed works on a strong rationality or selfishness assumption. In near future, we plan to extend the work to consider a 'bounded rationality'[9] model for humans. Also, our current model considers only explicitly quantifiable incentives. We realize that other than very few scenarios (e.g. second-life monetization [17]), explicit quantification of gains and costs is still difficult. As what happens with all nascent fields, no numerical data is readily available on costs and gains for social media contributions (e.g. wiki content provision, or youtube video sharing). While we had to study the current framework using numerical case studies, we want to undertake more work to better quantify such costs and gains.

Our current model considers tasks that only need contribution from one user and other contributions are redundant. We are working on extending the approach to consider cases which require $k$ different contributions or viewpoints (e.g. minimum 2 images required for stereoscopy or minimum 5 spam flags for post removal etc.). We are also considering a graded utility model wherein the value of each successive contribution gets lower but is still finite. Lastly, it would also be relevant to consider scenarios involving $j$ tasks, each needing $k$ contributions amongst the $N$ users.

We also intend to broaden the motivation factors to considered to include intrinsic motivation factors and concepts like Maslow's hierarchy and using them appropriately in the future models. Lastly, the enhancements obtained due to mechanism design were gained (amongst other reasons like optimality based incentive levels), because of the setting that benefits granted were 'virtual', while the costs and gains were 'real-world'. However, we feel this is indeed true in many social media environments like citizen-journalism, image-labeling, wiki-contributions etc.

While we admit, that our modeling is by no means perfect, this is meant to be a first step in drawing research interest towards this area. The value of this paper lies in providing food-for-thought to social media designers and developers charged with creating crowd-sourcing, media applications that require individual contributions to enhance the overall value of the application and its content.

\section{CONCLUSIONS}

In this work we have proposed a game-theoretic framework for studying user behavior and motivation patterns in social media networks. We have modeled users as rational selfish agents, and considered domain attributes like voluntary participation, virtual reward structure and public-sharing to model the dynamics of this interaction. We first studied the aspects of user-user interaction and used that to find the most appropriate contribution strategy from each user's perspective. The model created showed how the probability of task completion may decrease with large $N$ and made explicit the concepts like free-rider problem. We next studied the dynamics of system-user interaction, and showed how a system designer can design incentive mechanisms which help in improving the overall system performance, while possibly still benefiting the individual users.

\section{ACKNOWLEDGMENTS}

The authors would like to thank Ramarcha Kumar from Department of Economics, University of California, Irvine for multiple interactions on Game theoretic aspects of this work.

\section{REFERENCES}

[1] Google image labeler. http://images.google.com/imagelabeler/, Last accessed:07/06/2009.

[2] Microsoft photo-synth project. http://photosynth.net/, Last accessed:07/06/2009.

[3] New york times launches 'citizen journalism' sites. http://washingtonsquarepark.wordpress.com/2009/03/03/newyork-times-launches-citizen-journalism-sites/, Last accessed:07/06/2009.

[4] Wikipedia. http://www.wikipedia.org.

[5] C. Dellarocas. Reputation mechanism design in online trading environments with pure moral hazard. Information Systems Research, 16(2):209-230, June 2005.

[6] P. A. Diamond and J. A. Mirrlees. Optimal taxation and public production ii: Tax rules. The American Economic Review, 61(3):261-278, 1971.

[7] F. M. Harper, S. X. Li, Y. Chen, and J. A. Konstan. Social comparisons to motivate contributions to an online community. Persuasive Technology, 4744/2007, 2007.

[8] J. Howe. The rise of crowdsourcing. Wired Magazine, 14(6):1-4, 2006.

[9] D. Kahneman. Maps of bounded rationality: Psychology for behavioral economics. The American Economic Review, 93(5):1449-1475, 2003.

[10] O. Kim and M. Walker. The free rider problem: Experimental evidence. Public Choice, 43(1):3-24, 1984.

[11] P. Lévy. Collective intelligence: Mankind's emerging world in cyberspace. Perseus Books Cambridge, MA, USA, 1997.

[12] M. Maia, J. Almeida, and V. Almeida. Identifying user behavior in online social networks. In SocialNets '08: Proceedings of the 1st workshop on Social Network Systems, pages 1-6, 2008.

[13] M. Olson. The logic of collective action: Public goods and the theory of groups. Harvard Univ Press, 1971.

[14] M. J. Osborne. An Introduction to Game Theory. Oxford University Press, USA, 2003

[15] J. Schroer and G. Hertel. Voluntary Engagement in an Open Web-Based Encyclopedia: Wikipedians and Why They Do It. Media Psychology, 12(1):96-120, 2009.

[16] L. Sun and J. Vassileva. Social visualization encouraging participation in online communities. Groupware: Design, Implementation, and Use, 2006.

[17] D. Terdiman. The Entrepreneur's Guide to Second Life: Making Money in the Metaverse. Sybex, 2007.

[18] R. Vane. Advances in hypergame theory. In Workshop on Game Theoretic and Decision Theoretic Agents Conference on Autonomous Agents and Multi-Agent Systems, 2006.

[19] L. von Ahn. Games with a purpose. Computer, 39(6):92-94, June 2006. 